\title{
New Method for Simultaneous Quantification of 12 Ginsenosides in Red Ginseng Powder and Extract: In-house Method Validation
}

\author{
Gyo In*, Nam-Geun Ahn, Bong-Seok Bae, Sung-Tai Han, Kil-Bong Noh, and Cheon-Suk Kim \\ Analysis Research Team, R\&D Headquarters, Korea Ginseng Corporation, Daejeon 305-805, Korea
}

For quality control of components in Korean red ginseng powder and extract, a new method for simultaneous quantification of 12 ginsenosides ( $R g_{1}, R e, R f, R_{1}, \operatorname{Rg}_{2}[S], \operatorname{Rg}_{2}[R], R_{1}, R c, R_{2}, R d, \operatorname{Rg}_{3}[S]$, and $\operatorname{Rg}_{3}[R]$ ) was studied. Compared to the official method for quantification of marker substances (ginsenosides $\mathrm{Rg}_{1}$ and $\mathrm{Rb}_{1}$ ), the proposed methods were guaranteed by in-house method validation. Several criteria such as linearity, specificity, precision and accuracy were evaluated. For red ginseng powder, recovery (averaging 95\% to 105\%) was calculated, and analysis of variance was carried out to estimate the relative standard deviation $(0.20 \%$ to $2.12 \%)$. For red ginseng extract, the average recovery rate was $90 \%$ to $99 \%$ and the relative standard deviation was $0.39 \%$ to $2.40 \%$. These results indicate that the proposed method could be used in the laboratory for determination of 12 ginsenosides in red ginseng powder and extract. In addition, this method was found to be suitable for quality control of ginseng products and potentially offer time and cost benefits.

Keywords: Panax ginseng, Red ginseng, Ginsenosides, Method validation, Analysis, HPLC-photo diode array detector

\section{INTRODUCTION}

Ginseng (Panax ginseng Meyer) has long been considered as one of the most valuable medicinal herbs and is now used as a functional food and a raw material for pharmaceuticals. The efficacy of ginseng has been studied by many researchers [1]. For this reason, the market of "ginseng" especially "red ginseng" has been emerged continuously, more so than other functional food compounds [2]. The Korean government made a public announcement about a standard analysis method for determining the content of marker substances (ginsenosides $\mathrm{Rg}_{1}$ and $\mathrm{Rb}_{1}$ ) in ginseng product [3]. Additionally the Korea Food \& Drug administration announced that the standards and specification for ginseng content was changed from evaluating crude saponin contents to determining the sum of ginsenosides $\mathrm{Rg}_{1}$ and $\mathrm{Rb}_{1}$ [4].

For the analysis of ginsenosides, various analytical

(c) This is an Open Access article distributed under the terms of the Creative Commons Attribution Non-Commercial License (http://creativecommons.org/licenses/by-nc/3.0/) which permits unrestricted non-commercial use, distribution, and reproduction in any medium, provided the original work is properly cited. methods, such as TLC, GC, HPLC, capillary electrophoresis (CE), near infra-red spectroscopy (NIRS) were performed [5]. For NIRS, statistical algorithms such as principal component analysis and soft independent modeling of class analogy were applied to the data for quality control and distinction of ginseng and ginseng product [6]. CE is highly sensitive and fast, but there have been very few applications in which it was used for the analysis of ginsenosides due to the absence of a charge in its analytes [5]. In the beginning of 1980 when the analysis of ginsenosides by GC was developed, the analysis of ginsenosides by GC was usually performed directly on the trimethylsillyl derivatives of ginsenosides [5].

In 1978 HPLC was commercialized, and it is the most commonly used method for the analysis of ginsenosides in most studies on ginseng plant material and prepara-

Received 24 Jan. 2011, Revised 27 Feb. 2012, Accepted 27 Feb. 2012

*Corresponding author

E-mail: 20109042@kgc.or.kr

Tel: +82-42-870-3051, Fax: +82-42-870-3142 
tions, because of its speed, sensitivity and ability to analyze polar compounds. Different techniques have been used for the detection of ginsenosides such as UV, evaporative light scattering detection (ELSD), fluorescence fluorescence, and MS [1,5].

Fluorescence is one of the most sensitive detection methods in HPLC analysis. However, because ginsenosides do not contain suitable fluorescence chromophore they must be derivatized before detection, thus, fluorescence detection has been limited [5]. With the development of sophisticated ionization techniques, LC-MS techniques have been successfully applied for identification of trace amounts of ginsenosides and its metabolite analysis. This method was used as one of the most powerful verification tools in trace analysis and biochemistry fields $[7,8]$.

HPLC-UV and HPLC-ELSD methods have been widely used for ginsenosides analysis and some modification was performed by many researchers $[9,10]$. Although both methods have advantages and disadvantages for ginsenosides analysis, HPLC-UV is more widely employed of the two because the detection method is more common. HPLC-UV was employed as a standard method for testing ginseng and ginseng products.

In this paper, a rapid and simple pretreatment method for simultaneous determination of 12 ginsenosides including marker substances of ginseng product (ginsenosides $\mathrm{Rg}_{1}$ and $\mathrm{Rb}_{1}$ ) was studied. Method validation was carried out by comparison of some parameters such as precision and recovery. As a result, the proposed method may be used for quality control of ginseng products.

\section{MATERIALS AND METHODS}

\section{Materials and equipments}

All distilled water used in the experiment were purified by Milli-Q water system (Millipore, Bedford, MA, USA) and the resistance value was measured as $18 \mathrm{M} \Omega$ prior to use. HPLC analysis was carried out by using an Alliance 2695 HPLC system (Waters, Milford, MA, USA) and 2996 photo diode array detector (Waters) as shown in Table 1.

Ginsenoside $\mathrm{Rg}_{1}, \mathrm{Re}, \mathrm{Rf}, \mathrm{Rh}_{1}, \mathrm{Rb}_{1}, \mathrm{Rc}, \mathrm{Rb}_{2}, \mathrm{Rd}$, $\mathrm{Rg}_{3}(\mathrm{~S})$, and $\mathrm{Rg}_{3}(\mathrm{R})$ were purchased from Chromadex Co. (Irvine, CA, USA) and ginsenoside $\mathrm{Rg}_{2}(\mathrm{~S})$ and $\mathrm{Rg}_{2}(\mathrm{R})$ were obtained from Embo Lab. (Seoul, Korea). All reagents used in the experiment were guaranteed reagent grade, and HPLC-grade acetonitrile and methanol were purchased from Merck (Darmstadt, Germany). Red ginseng powder (lot no. 1019008) and red ginseng extract (lot no. 2019119) was purchased from the local health food
Table 1. Instrumental conditions for ginsenosides analysis

\begin{tabular}{cccc}
\hline Time $(\mathrm{min})$ & Flow $(\mathrm{mL} / \mathrm{min})$ & $\mathrm{A} \%, \mathrm{ACN}$ & $\mathrm{B} \%, \mathrm{DW}$ \\
\hline Initial & 1.6 & 80 & 20 \\
40 & 1.6 & 68 & 32 \\
55 & 1.6 & 50 & 50 \\
70 & 1.6 & 35 & 65 \\
71 & 1.6 & 10 & 90 \\
81 & 1.6 & 80 & 20 \\
90 & 1.6 & 80 & 20 \\
\hline
\end{tabular}

Column: Supelco Discovery C18, $5 \mu \mathrm{m}, 250 \times 4.6 \mathrm{~mm}$; injection volume: $20 \mu \mathrm{L}$; column temperature: $40 \pm 2^{\circ} \mathrm{C}$; detector: Waters 2998 photo diode array detector, $203 \pm 0.2 \mathrm{~nm}$.

$\mathrm{ACN}$, acetonitrile; DW, deionized water.

shop (Korea Ginseng Corporation, Daejeon, Korea).

\section{Method validation}

To obtain validity and similarity between the proposed method and the official method, the analytical results of ginsenosides were compared to one other in accordance with International Conference on Harmonisation (ICH) guideline $[11,12]$. The precision and accuracy of the analytical methods were determined by comparison of 3 replicates at 3 different concentrations using the same sample and conditions. More details are provided in the section that follows.

\section{Analytical procedure for red ginseng powder Official method}

One gram of red ginseng powder was weighed in a round bottom flask, and $50 \mathrm{~mL}$ of $50 \% \mathrm{MeOH}$ was added. The solution was then refluxed for $1 \mathrm{~h}$. After cooling, the sample was filtered (Whatman no. 4; Whatman, Clifton, NJ, USA). The supernatant solution was collected, and the residue was treated as the same procedure. The collected liquids were combined and evaporated (Butchi, Flawil, Switzerland) under reduced pressure at a temperature not exceeding $60^{\circ} \mathrm{C}$ and the residue was dissolved in $25 \mathrm{~mL}$ of solvent (deionized water:acetonitrile $=8: 2$ ). Then, this solution was filtered $(0.2 \mu \mathrm{m}$; Acrodisk, Port Washington, NY, USA) and injected into the HPLC system.

\section{Proposed method}

A half of gram of red ginseng powder was weighed in a centrifugal tube $(15 \mathrm{~mL}$, polypropylene-single use; $\mathrm{Bi}$ oLogix, USA) and shaken vigorously after the addition of $10 \mathrm{~mL}$ of $50 \% \mathrm{MeOH}$. Extraction was performed in an ultrasonic cleaner $(60 \mathrm{~Hz}$; Wiseclean, Seoul, Korea) for $30 \mathrm{~min}$. After ultrasonic extraction, centrifugal separation (Legend Mach 1.6R; Thermo, Frankfurt, Germany) 
was performed for $10 \mathrm{~min}$, at 3,000 rpm. The resulting supernatant solution was filtered $(0.2 \mu \mathrm{m}$, Acrodisk $)$ and injected into the HPLC system.

\section{Analytical procedure for red ginseng extract Official method}

Two gram of red ginseng extract was weighed in a beaker, and $20 \mathrm{~mL}$ of deionized water was added. After sitting at room temperature for $1 \mathrm{~h}$, the diluted sample was dissolved in $50 \mathrm{~mL}$ of deionized water. The solution was filtered $(0.2 \mu \mathrm{m}$, Acrodisk) and injected into the HPLC system.

\section{Proposed method}

Two gram of red ginseng extract were weighed in a beaker, and $25 \mathrm{~mL}$ of deionized water was added. After sitting

A

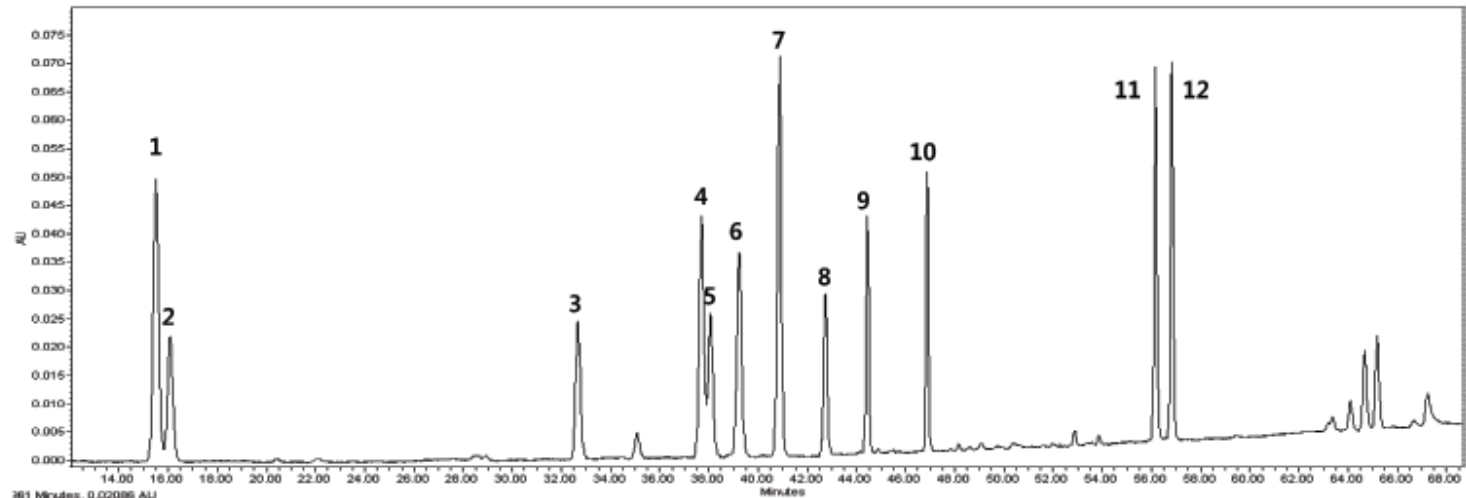

B

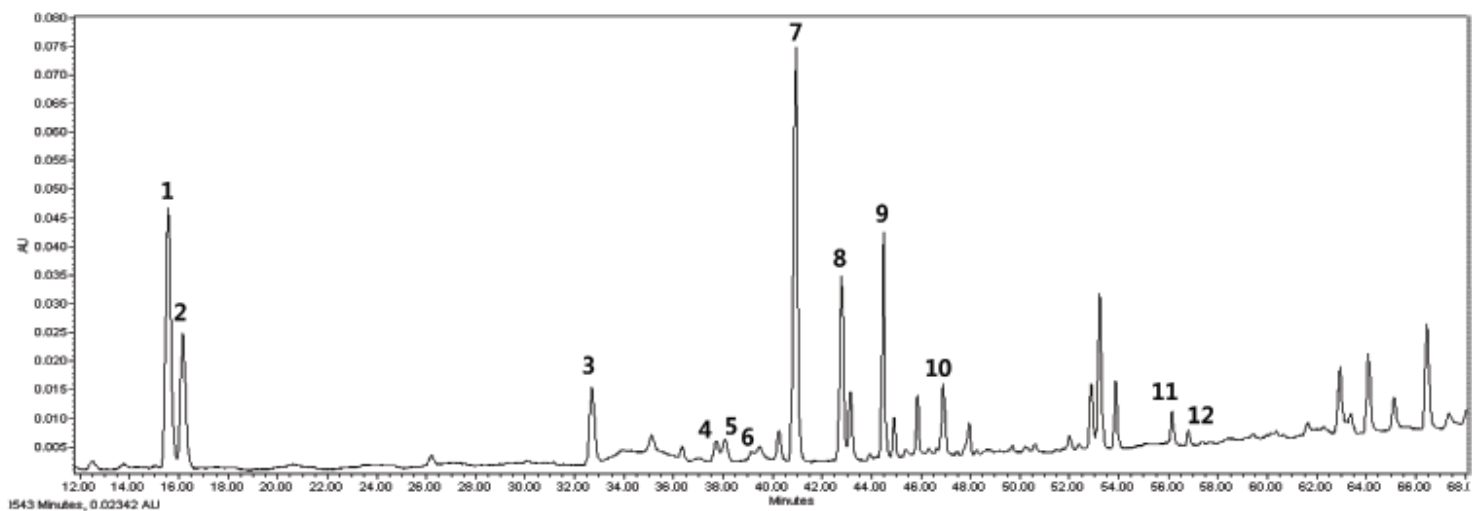

C

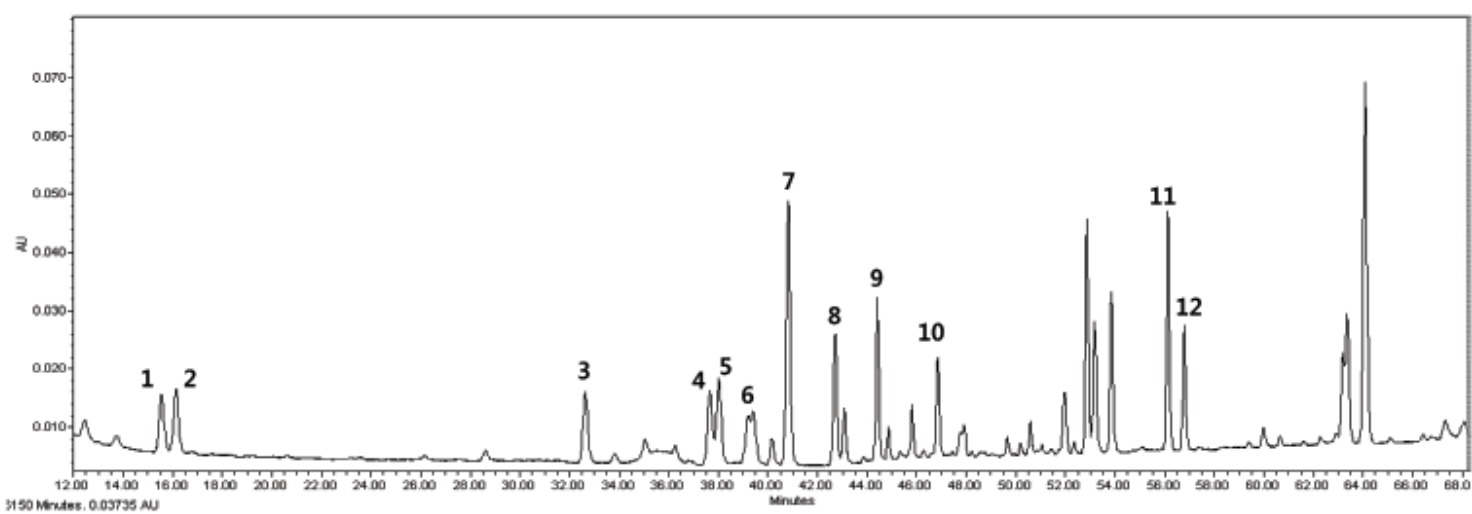

Fig. 1. Representative HPLC chromatogram of mixed standards (A), red ginseng powder (B), and red ginseng extract (C). $1, \mathrm{Rg}_{1}(15.53 \mathrm{~min}) ; 2$, $\operatorname{Re}(16.12 \mathrm{~min}) ; 3, \mathrm{Rf}(32.66 \mathrm{~min}) ; 4, \mathrm{Rh}_{1}(37.67 \mathrm{~min}) ; 5, \mathrm{Rg}_{2}(\mathrm{~S})$ (38.04 min); 6, $\mathrm{Rg}_{2}(\mathrm{R})$ (39.22 min); 7, $\mathrm{Rb}_{1}(40.89 \mathrm{~min}) ; 8, \mathrm{Rc}(42.74 \mathrm{~min}) ; 9, \mathrm{Rb}$ (44.44 min); 10, Rd (46.86 min); 11, $\mathrm{Rg}_{3}(\mathrm{~S})$ (56.15 min); 12, $\mathrm{Rg}_{3}(\mathrm{R})(56.80 \mathrm{~min})$. 
Table 2. Linearity of calibration curve for ginsenosides

\begin{tabular}{|c|c|c|c|c|}
\hline Ginsenoside & Calibration curve $^{1)}$ & $\mathrm{R}^{2}$ & $\mathrm{LOD}(\mu \mathrm{g} / \mathrm{mL})$ & $\mathrm{LOQ}(\mu \mathrm{g} / \mathrm{mL})$ \\
\hline $\mathrm{Rg}_{1}$ & $\mathrm{Y}=4.52 * 10^{3} \mathrm{X}-4.25 * 10^{3}$ & 0.999933 & 1.74 & 5.80 \\
\hline $\operatorname{Re}$ & $\mathrm{Y}=3.82 * 10^{3} \mathrm{X}+8.88 * 10^{2}$ & 0.999961 & 1.71 & 5.70 \\
\hline $\mathrm{Rf}$ & $\mathrm{Y}=3.92 * 10^{3} \mathrm{X}-9.62 * 10^{2}$ & 0.999958 & 1.08 & 3.59 \\
\hline $\mathrm{Rh}_{1}$ & $\mathrm{Y}=6.45 * 10^{3} \mathrm{X}-7.48 * 10^{3}$ & 0.999970 & 0.84 & 2.82 \\
\hline $\operatorname{Rg}_{2}(\mathrm{~S})$ & $\mathrm{Y}=5.03 * 10^{3} \mathrm{X}-1.68 * 10^{4}$ & 0.999932 & 0.36 & 1.21 \\
\hline $\operatorname{Rg}_{2}(\mathrm{R})$ & $\mathrm{Y}=5.30 * 10^{3} \mathrm{X}+1.16 * 10^{4}$ & 0.999978 & 0.48 & 1.58 \\
\hline $\mathrm{Rb}_{1}$ & $\mathrm{Y}=3.33 * 10^{3} \mathrm{X}-4.70 * 10^{3}$ & 0.999937 & 0.90 & 2.97 \\
\hline $\mathrm{Rc}$ & $\mathrm{Y}=3.69 * 10^{3} \mathrm{X}-1.12 * 10^{4}$ & 0.999937 & 1.92 & 6.37 \\
\hline $\mathrm{Rb}_{2}$ & $\mathrm{Y}=3.55 * 10^{3} \mathrm{X}-5.54 * 10^{3}$ & 0.999937 & 1.77 & 5.86 \\
\hline $\mathrm{Rd}$ & $\mathrm{Y}=4.12 * 10^{3} \mathrm{X}-5.72 * 10^{3}$ & 0.999924 & 1.47 & 4.85 \\
\hline $\operatorname{Rg}_{3}(\mathrm{~S})$ & $\mathrm{Y}=4.18 * 10^{3} \mathrm{X}-3.41 * 10^{3}$ & 0.999910 & 0.33 & 1.12 \\
\hline $\operatorname{Rg}_{3}(\mathrm{R})$ & $\mathrm{Y}=5.71 * 10^{3} \mathrm{X}-6.03 * 10^{3}$ & 0.999919 & 0.20 & 0.66 \\
\hline
\end{tabular}

LOD, limit of detection; LOQ, limit of quantification.

${ }^{1)} Y$ : peak area; $X$ : amount of ginsenoside $(\mu \mathrm{g} / \mathrm{mL})$.

at room temperature for $1 \mathrm{~h}$, the diluted sample was transferred in a $50 \mathrm{~mL}$ volumetric flask where the volume was brought up to $50 \mathrm{~mL}$ by adding $\mathrm{MeOH}$. Extraction was performed in an ultrasonic cleaner $(60 \mathrm{~Hz}$, Wiseclean) for $30 \mathrm{~min}$. Then, the solution was filtered $(0.2 \mu \mathrm{m}$, Acrodisk $)$ and injected into the HPLC system.

\section{RESULTS AND DISCUSSION}

\section{Specificity, linearity, and limit of detection}

The specificity of individual ginsenosides was confirmed by demonstrating a sufficient separation of the substance present in the sample matrix. As shown in Fig. 1, sample chromatogram compared with that of the standard solution was sufficient to confirm the specificity of ginsenosides. In other words appropriate separation means that there is adequate resolution between the analyte peaks and the impurity and placebo peaks that need not be separated from each other [12].

A linear dependence of the signal and the analyte concentration is the most convenient indicator of sample quality or purity and is widely used in pharmaceutical analysis [12]. The standard solution containing 20 to $150 \mu \mathrm{g} / \mathrm{mL}$ of each ginsenosides were injected into the HPLC. Calibration curves were plotted as the peak area versus the amount of each analyte. The linearity was evaluated by linear regression analysis, which is calculated by the least squares regression method. The limit of quantification under the present chromatographic conditions was determined on the basis of the response at a signal to noise ratio of 10 . The linearity of calibration curve for 12 ginsenosides was demonstrated in Table 2.
Table 3. Ginsenosides amount $(\mathrm{mg} / \mathrm{g})$ and precision result using red ginseng powder (means of 9 determinations over 3 concentration levels)

\begin{tabular}{lccc}
\hline Ginsenoside & $\begin{array}{c}\text { Official method } \\
(\mathrm{mg} / \mathrm{g})\end{array}$ & $\begin{array}{c}\text { Proposed method } \\
(\mathrm{mg} / \mathrm{g})\end{array}$ & $\begin{array}{c}\text { Bias } \\
(\%)\end{array}$ \\
\hline $\mathrm{Rg}_{1}$ & $2.966 \pm 0.012$ & $3.260 \pm 0.009$ & 9.0 \\
$\mathrm{Re}$ & $1.709 \pm 0.011$ & $1.907 \pm 0.012$ & 10.4 \\
$\mathrm{Rf}$ & $0.955 \pm 0.014$ & $1.052 \pm 0.005$ & 9.2 \\
$\mathrm{Rh}_{1}$ & $0.095 \pm 0.002$ & $0.135 \pm 0.002$ & 29.6 \\
$\mathrm{Rg}_{2}(\mathrm{~S})$ & $0.138 \pm 0.001$ & $0.188 \pm 0.001$ & 26.6 \\
$\mathrm{Rg}_{2}(\mathrm{R})$ & $0.121 \pm 0.001$ & $0.110 \pm 0.001$ & -10.0 \\
$\mathrm{Rb}_{1}$ & $5.022 \pm 0.007$ & $5.301 \pm 0.022$ & 5.3 \\
$\mathrm{Rc}$ & $1.958 \pm 0.024$ & $2.042 \pm 0.018$ & 4.1 \\
$\mathrm{Rb}_{2}$ & $1.832 \pm 0.023$ & $1.937 \pm 0.006$ & 5.4 \\
$\mathrm{Rd}$ & $0.479 \pm 0.003$ & $0.579 \pm 0.002$ & 17.3 \\
$\mathrm{Rg}_{3}(\mathrm{~S})$ & $0.214 \pm 0.001$ & $0.208 \pm 0.001$ & -2.9 \\
$\mathrm{Rg}_{3}(\mathrm{R})$ & $0.084 \pm 0.001$ & $0.058 \pm 0.001$ & -44.8 \\
\hline
\end{tabular}

\section{Precision and accuracy for analysis of red ginseng powder}

The precision of the proposed method was tested by comparing results from this methods with results obtained from using the official method. Thus 9 data points spanning 3 concentration levels were obtained by both methods. As shown in Table 3, the relative standard deviation of 3 results was below $3 \%$, which was sufficient to prove the validity of the proposed method. However, some trends in these variations were observed between the proposed and official analytical results. The analytical results of the official method for major ginsenosides such as $\mathrm{Rg}_{1}, \mathrm{Re}, \mathrm{Rf}, \mathrm{Rb}_{1}, \mathrm{Rc}$, and $\mathrm{Rb}_{2}$ were below that of the proposed method. This is because thermal degradation 
Table 4. Recovery and RSD values of red ginseng powder ( 9 determinations over 3 concentration levels)

\begin{tabular}{|c|c|c|c|c|c|}
\hline \multirow{2}{*}{ Component } & \multicolumn{2}{|c|}{ Official method } & \multicolumn{2}{|c|}{ Proposed method } & \multirow{2}{*}{ Bias $(\%$} \\
\hline & Recovery $(\%)^{1)}$ & RSD & Recovery $(\%)^{1)}$ & RSD & \\
\hline $\operatorname{Rg}_{1}$ & 102.8 & 2.82 & 102.8 & 5.71 & 0.0 \\
\hline $\operatorname{Re}$ & 98.1 & 3.17 & 101.6 & 5.26 & 3.4 \\
\hline $\mathrm{Rf}$ & 101.0 & 5.66 & 103.6 & 5.07 & 2.5 \\
\hline $\mathrm{Rh}_{1}$ & 82.5 & 9.92 & 103.6 & 2.87 & 20.4 \\
\hline $\operatorname{Rg}_{2}(\mathrm{~S})$ & 78.1 & 5.83 & 97.2 & 5.10 & 19.6 \\
\hline $\mathrm{Rg}_{2}(\mathrm{R})$ & 101.1 & 5.79 & 105.2 & 2.60 & 3.9 \\
\hline $\mathrm{Rb}_{1}$ & 95.5 & 6.83 & 101.9 & 4.48 & 6.2 \\
\hline $\mathrm{Rc}$ & 98.0 & 6.67 & 101.9 & 3.46 & 3.8 \\
\hline $\mathrm{Rb}_{2}$ & 99.4 & 6.82 & 102.4 & 4.85 & 2.9 \\
\hline $\mathrm{Rd}$ & 98.3 & 8.10 & 99.5 & 4.61 & 1.2 \\
\hline $\operatorname{Rg}_{3}(\mathrm{~S})$ & 97.3 & 5.64 & 95.0 & 4.90 & 2.5 \\
\hline $\operatorname{Rg}_{3}(\mathrm{R})$ & 96.2 & 6.21 & 96.8 & 4.49 & 0.7 \\
\hline
\end{tabular}

$\mathrm{RSD}$, relative standard deviation.

${ }^{1)}$ Mean value of 9 determinations over 3 concentration levels.

occurs in the glycosidic linkage at carbon 20 in the ginsenoside backbone. In other words, major ginsenosides were transformed to other ginsenosides by thermal energy during reflux. Minor ginsenosides such as $\mathrm{Rh}_{1}, \mathrm{Rg}_{2}$, and $\mathrm{Rg}_{3}$ showed a wider range of variation ( $17 \%$ to $\left.45 \%\right)$ because of the random errors that more seriously affected the trace amount analysis.

The accuracy of the proposed method was tested by spiking experiments for recovery investigations. Thus crude saponin fractions were spiked into the analytical samples for recovery tests of the 12 ginsenosides. As shown in Table 4, the statistical values for both methods were good. However, with regards to the precision of these experiments, the results from the official method demonstrated poorer recovery values than proposed method. It should be confirmed that the proposed method was more suitable for simultaneous determination of ginsenosides than the official method because the official method focuses primarily on quantitative analysis of ginsenosides $\mathrm{Rg}_{1}$ and $\mathrm{Rb}_{1}$.

\section{Precision and accuracy data for red ginseng ex- tract}

The validation of analytical methods for the red ginseng extract was also performed to evaluate the red ginseng powder. The precision of the 9 data point spanning 3 different concentrations are shown in Table 5, and Table 6 reports the accuracy of these experiments. The variability of data from these experiments was below 3\% relative standard deviation (RSD), suggesting high preci-
Table 5. Ginsenosides amount $(\mathrm{mg} / \mathrm{g})$ and precision result using red ginseng extract (means of 9 determinations over 3 concentration levels)

\begin{tabular}{lccc}
\hline Ginsenoside & $\begin{array}{c}\text { Official method } \\
(\mathrm{mg} / \mathrm{g})\end{array}$ & $\begin{array}{c}\text { Proposed method } \\
(\mathrm{mg} / \mathrm{g})\end{array}$ & Bias $(\%)$ \\
\hline $\mathrm{Rg}_{1}$ & $0.835 \pm 0.012$ & $0.867 \pm 0.011$ & 3.7 \\
$\mathrm{Re}$ & $1.059 \pm 0.014$ & $1.110 \pm 0.014$ & 4.6 \\
$\mathrm{Rf}$ & $1.167 \pm 0.006$ & $1.287 \pm 0.013$ & 9.3 \\
$\mathrm{Rh}_{1}$ & $0.770 \pm 0.004$ & $0.877 \pm 0.011$ & 12.2 \\
$\mathrm{Rg}_{2}(\mathrm{~S})$ & $1.134 \pm 0.010$ & $1.297 \pm 0.006$ & 11.3 \\
$\mathrm{Rg}_{2}(\mathrm{R})$ & $0.537 \pm 0.011$ & $0.598 \pm 0.012$ & 10.2 \\
$\mathrm{Rb}_{1}$ & $4.109 \pm 0.058$ & $4.278 \pm 0.039$ & 4.0 \\
$\mathrm{Rc}$ & $1.775 \pm 0.028$ & $1.896 \pm 0.045$ & 6.4 \\
$\mathrm{Rb}_{2}$ & $1.698 \pm 0.025$ & $1.790 \pm 0.007$ & 5.1 \\
$\mathrm{Rd}$ & $1.001 \pm 0.018$ & $1.072 \pm 0.005$ & 6.6 \\
$\mathrm{Rg}_{3}(\mathrm{~S})$ & $1.948 \pm 0.028$ & $2.215 \pm 0.049$ & 12.1 \\
$\mathrm{Rg}_{3}(\mathrm{R})$ & $0.797 \pm 0.004$ & $0.674 \pm 0.008$ & -18.2 \\
\hline
\end{tabular}

sion and repeatability. In terms of accuracy, the recovery of ginsenosides was $90 \%$ to $105 \%$, with respect to the spiked amount. Therefore, both methods were appropriate based on the accuracy of the data.

The analytical method was recognized as a valid process for ginseng analysis when high precision and repeatability of the data was demonstrated (5.3\% to $7.3 \%$ RSD) as well as a high degree of accuracy of the data $(80 \%$ to $107 \%$ of recovery). These criteria were set based on analyte content of 10 to $100 \mathrm{ug} / \mathrm{mL}$ in samples. Based on these criteria, the official method was inappropriate for analysis of trace amounts of ginsenosides $\mathrm{Rh}_{1}, \mathrm{Rg}_{2}, \mathrm{Rg}_{3}$ 
Table 6. Recovery and RSD values of red ginseng extract (9 determinations over 3 concentration levels)

\begin{tabular}{|c|c|c|c|c|c|}
\hline \multirow{2}{*}{ Component } & \multicolumn{2}{|c|}{ Official method } & \multicolumn{2}{|c|}{ Proposed method } & \multirow{2}{*}{ Bias (\%) } \\
\hline & Recovery $(\%)^{1)}$ & RSD & Recovery $(\%)^{1)}$ & RSD & \\
\hline $\operatorname{Rg}_{1}$ & 95.2 & 3.51 & 93.6 & 2.11 & 1.7 \\
\hline $\mathrm{Re}$ & 97.5 & 3.84 & 95.0 & 2.43 & 2.6 \\
\hline $\mathrm{Rf}$ & 95.8 & 4.21 & 92.3 & 1.53 & 3.9 \\
\hline $\mathrm{Rh}_{1}$ & 98.7 & 8.53 & 96.5 & 2.85 & 2.3 \\
\hline $\operatorname{Rg}_{2}(\mathrm{~S})$ & 95.8 & 5.99 & 99.1 & 7.72 & 3.3 \\
\hline $\mathrm{Rg}_{2}(\mathrm{R})$ & 93.2 & 3.40 & 96.4 & 9.41 & 3.2 \\
\hline $\mathrm{Rb}_{1}$ & 91.4 & 2.57 & 91.3 & 1.72 & 0.1 \\
\hline $\mathrm{Rc}$ & 97.0 & 5.18 & 92.5 & 1.41 & 4.9 \\
\hline $\mathrm{Rb}_{2}$ & 97.1 & 4.13 & 90.6 & 1.68 & 7.1 \\
\hline $\mathrm{Rd}$ & 96.9 & 3.94 & 92.9 & 3.05 & 4.3 \\
\hline $\operatorname{Rg}_{3}(\mathrm{~S})$ & 100.9 & 4.42 & 92.2 & 3.15 & 9.5 \\
\hline $\operatorname{Rg}_{3}(\mathrm{R})$ & 105.4 & 3.47 & 96.5 & 4.27 & 9.3 \\
\hline
\end{tabular}

$\mathrm{RSD}$, relative standard deviation.

${ }^{1)}$ Mean value of 9 determinations over 3 concentration levels.

and the proposed method was more suitable for simultaneous determination of ginsenosides.

In this paper, methods to perform simultaneous analysis of multiple ginsensides in red ginseng powder and red ginseng extract were evaluated. Validation of the proposed method was performed with guidance from the ICH guidelines.

Both methods were valid for analysis of ginsenosides $\mathrm{Rg}_{1}$ and $\mathrm{Rb}_{1}$ as a marker substance. However, judging from the low precision and accuracy, the official method was not suitable for determining trace amounts of ginsenosides in red ginseng powder or red ginseng extract. Therefore the proposed method may be applied for determination of ginsenoside content in ginseng products for quality control. The application of this method may have additional benefit of saving time and labor costs.

\section{REFERENCES}

1. Christensen LP. Ginsenosides chemistry, biosynthesis, analysis, and potential health effects. Adv Food Nutr Res 2009;55:1-99.
2. Park CK, Kwak YS, Hwang MS, Kim SC, Do JH. Trends and prospect of ginseng products in market health functional food. Food Sci Ind 2007;40:30-45.

3. Korean Agency for Technology and Standards. Ginseng and ginseng products - determination of ginsenoside ( $\mathrm{Rg} 1$, $\mathrm{Rb}_{1}$ ) contents - method by high performance liquid chromatography. KSH2153. 2008 Mar 26.

4. Korea Food \& Drug Administration. Health functional food code. Seoul: Korea Food \& Drug Administration, 2010.

5. Fuzzati N. Analysis methods of ginsenosides. J Chromatogr B Analyt Technol Biomed Life Sci 2004;812:119133.

6. Yap KY, Chan SY, Lim CS. Infrared-based protocol for the identification and categorization of ginseng and its products. Food Res Int 2007;40:643-652.

7. Ji HY, Lee HW, Kim HK, Kim HH, Chang SG, Sohn DH, Kim J, Lee HS. Simultaneous determination of ginsenoside $\mathrm{Rb}_{1}$ and $\mathrm{Rg}_{1}$ in human plasma by liquid chromatography-mass spectrometry. J Pharm Biomed Anal 2004;35:207-212.

8. Dan M, Su M, Gao X, Zhao T, Zhao A, Xie G, Qiu Y, Zhou M, Liu Z, Jia W. Metabolite profiling of Panax notoginseng using UPLC-ESI-MS. Phytochemistry 2008;69:2237-2244.

9. Shi Y, Sun C, Zheng B, Li Y, Wang Y. Simultaneous determination of nine ginsenosides in functional foods by high performance liquid chromatography with diode array detector detection. Food Chem 2010;123:1322-1327.

10. Kim SN, Ha YW, Shin H, Son SH, Wu SJ, Kim YS. Simultaneous quantification of 14 ginsenosides in Panax ginseng C.A. Meyer (Korean red ginseng) by HPLCELSD and its application to quality control. J Pharm Biomed Anal 2007;45:164-170.

11. Taverniers I, Loose MD, Bockstaele EV. Trends in quality in the analytical laboratory. II. Analytical method validation and quality assurance. TrAC Trends Anal Chem 2004;23:535-552.

12. Ermer J. Validation in pharmaceutical analysis. Part I: an integrated approach. J Pharm Biomed Anal 2001;24:755767. 